\title{
GENERALIZATION OF EXTENDED BETA FUNCTION, HYPERGEOMETRIC AND CONFLUENT HYPERGEOMETRIC FUNCTIONS
}

\author{
Dong Myung Lee, Arjun K. Rathie, Rakesh K. Parmar and \\ YONG SUP KIM*
}

\begin{abstract}
The main object of this paper is to present generalization of extended beta function, extended hypergeometric and confluent hypergeometric function introduced by Chaudhry et al. and obtained various integral representations, properties of beta function, Mellin transform, beta distribution, differentiation formulas, transform formulas, recurrence relations, summation formula for these new generalization.
\end{abstract}

\section{Introduction}

We first recall the Euler's beta function [9], which has the integral representation

$$
B(x, y)=\int_{0}^{1} t^{x-1}(1-t)^{y-1} d t, \quad \Re e(x)>0, \Re e(y)>0 .
$$

Euler generalized the fractional function from the domain of natural numbers to the gamma function and defined Euler's gamma function [9]

$$
\Gamma(n)=\int_{0}^{\infty} t^{n-1} e^{-t} d t, \quad \Re e(n)>0 .
$$

The classical Gauss hypergeometric function (GHF) defined by [9]

Received March 15, 2011. Accepted April 27, 2011.

2000 Mathematics Subject Classification. 33B15; 33C05; 33C15.

Key words and phrases. Extended beta function; Entended hypergeometric function, Extended confluent hypergeometric function; Mellin transform, Beta distribution.

This paper was supported by Wonkwang University in 2011.

*Corresponding author. 


$$
{ }_{2} F_{1}(a, b ; c ; z)=\sum_{n=0}^{\infty} \frac{(a)_{n}(b)_{n}}{(c)_{n}} \frac{z^{n}}{n !} .
$$

The GHF can be given an integral representation as follows [9, p. 85]

$$
{ }_{2} F_{1}(a, b ; c ; z)=\frac{1}{B(b, c-b)} \int_{0}^{1} t^{b-1}(1-t)^{c-b-1}(1-z t)^{-a} d t,
$$

where $|\arg (1-z)|<\pi, \Re e(c)>\Re e(b)>0$.

We can also write the GHF in terms of the beta function (1.1) as follows [1]:

$$
{ }_{2} F_{1}(a, b ; c ; z)=\sum_{n=0}^{\infty} \frac{B(b+n, c-b)}{B(b, c-b)}(a)_{n} \frac{z^{n}}{n !},
$$

where $|z|<1, \Re e(c)>\Re e(b)>0$. The confluent hypergeometric function $(\mathrm{CHF})$ defined by

$$
{ }_{1} F_{1}(b ; c ; z)=\sum_{n=0}^{\infty} \frac{(b)_{n}}{(c)_{n}} \frac{z^{n}}{n !} .
$$

The CHF can be given an integral representation as follows [9, p. 85] (1.5)

$$
{ }_{1} F_{1}(b ; c ; z)=\frac{1}{B(b, c-b)} \int_{0}^{1} t^{b-1}(1-t)^{c-b-1} e^{z t} d t, \quad \Re e(c)>\Re e(b)>0,
$$

The confluent hypergeometric function $(\mathrm{CHF})$ can be written in terms of the beta function to give

$$
{ }_{1} F_{1}(b ; c ; z)=\sum_{n=0}^{\infty} \frac{B(b+n, c-b)}{B(b, c-b)} \frac{z^{n}}{n !},
$$

where $\Re e(c)>\Re e(b)>0$. In recent years, several extensions of the wellknown special functions have been considered by some authors [1-7]. In 1994, Chaudhry and Zubair [1] have introduced the following extension of gamma function

$$
\Gamma_{p}(x)=\int_{0}^{\infty} t^{x-1} \exp \left[-t-\frac{p}{t}\right] d t \quad \Re e(p)>0 .
$$

In 1997, Chaudhry et al. [2] introduced the following extension of Euler's beta function

$$
B(x, y ; p)=\int_{0}^{1} t^{x-1}(1-t)^{y-1} \exp \left[-\frac{p}{t(1-t)}\right] d t
$$


where $\Re e(p)>0, \Re e(x)>0$, and $\Re e(y)>0$ and they proved that this extension has connection with the Macdonald, Error and Whittakers function. It is clearly seen that when $p=0,(1.7)$ and (1.8) reduces to original gamma function and beta function. Recently, Chaudhry et al. [6] used $B(x, y ; p)$ to extend the hypergeometric functions and confluent hypergeometric functions as follows;

$$
F_{p}(a, b ; c ; z)=\sum_{n=0}^{\infty} \frac{B(b+n, c-b ; p)}{B(b, c-b)}(a)_{n} \frac{z^{n}}{n !},
$$

where $p \geq 0,|z|<1, \Re e(c)>\Re e(b)>0$.

$$
\Phi_{p}(b ; c ; z)=\sum_{n=0} \frac{B(b+n, c-b ; p)}{B(b, c-b)} \frac{z^{n}}{n !},
$$

where $p \geq 0, \Re e(c)>\Re e(b)>0$ and gave the integral representation

$F_{p}(a, b ; c ; z)=\frac{1}{B(b, c-b)} \int_{0}^{1} t^{b-1}(1-t)^{c-b-1}(1-z t)^{-a} \exp \left[-\frac{p}{t(1-t)}\right] d t$,

where $p \geq 0$ and $|\arg (1-z)|<\pi, \Re e(c)>\Re e(b)>0$.

$$
\Phi_{p}(b ; c ; z)=\frac{1}{B(b, c-b)} \int_{0}^{1} t^{b-1}(1-t)^{c-b-1} \exp \left[z t-\frac{p}{t(1-t)}\right] d t,
$$

where $p \geq 0, \Re e(c)>\Re e(b)>0$. They called these functions extended Gauss hypergeometric function (EGHF) and extended confluent hypergeometric function (ECHF), respectively. They have obtained the integral representations, beta distribution, various properties of differentiation formulas, Mellin transform, transformation formulas, recurrence relations, summation and asymptotic formulas for these newly defined function.

Clearly, when $p=0,(1.9),(1.10),(1.11)$ and (1.12) reduces to usual GHF and CHF. In this paper, we consider the following generalizations of Euler's beta functions as

$$
B(x, y ; p ; m)=\int_{0}^{1} t^{x-1}(1-t)^{y-1} \exp \left[-\frac{p}{t^{m}(1-t)^{m}}\right] d t,
$$

where $\Re e(p)>0, m>0$. Clearly when $m=1$, equation (1.13) reduces to Chaudhry et al. [2] extended beta function (EBF) and $p=0$, it reduces to usual Euler's beta function [9]. 
In section 2, different integral representation and some properties of new generalized Euler's beta function are obtained. Additionally, relations of new generalized beta functions are discussed.

In section 6 , we generalize the hypergeometric function and confluent hypergeometric function using $B(x, y ; p ; m)$ and obtain the integral representations of new generalized Gauss's hypergeometric function. Further we obtain the differentiation formulas, Mellin transforms, transformation formulas, recurrence relations, summation formulas for these newly defined hypergeometric function and confluent hypergeometric functions.

\section{Integral representation of new generalized beta function}

Theorem 2.1.

$$
\int_{0}^{\infty} p^{s-1} B(x, y ; p ; m) d p=\Gamma(s) B(x+m s, y+m s),
$$

where $\Re e(p)>0, \Re e(s)>0, \Re e(x+m s)>0, \Re e(y+m s)>0$ and $m>0$.

Proof. Multiplying (1.13) by $p^{s-1}$ and integrating with respect to $p$ from $p=0$ to $p=\infty$, we get

$$
\begin{aligned}
& \int_{0}^{\infty} p^{s-1} B(x, y ; p ; m) d p \\
& =\int_{0}^{\infty} p^{s-1}\left(\int_{0}^{1} t^{x-1}(1-t)^{y-1} \exp \left(-\frac{p}{t^{m}(1-t)^{m}}\right) d t\right) d p .
\end{aligned}
$$

The order of integral in (2.2) can be interchanged because of the uniform convergence of the integral. Therefore, we have

$$
\begin{aligned}
& \int_{0}^{\infty} p^{s-1} B(x, y ; p ; m) d p \\
& =\int_{0}^{1} t^{x-1}(1-t)^{y-1}\left[\int_{0}^{\infty} p^{s-1} \exp \left(-\frac{p}{t^{m}(1-t)^{m}}\right) d p\right] d t .
\end{aligned}
$$


Using definition of gamma function in (2.3), we have

$$
\begin{aligned}
\int_{0}^{\infty} p^{s-1} B(x, y ; p ; m) d p & =\int_{0}^{1} t^{x-1}(1-t)^{y-1}\left(t^{m s}(1-t)^{m s} \Gamma(s)\right) d t \\
& =\Gamma(s) \int_{0}^{1} t^{x+m s-1}(1-t)^{y+m s-1} d t \\
& =\Gamma(s) B(x+m s, y+m s) .
\end{aligned}
$$

This completes the proof of the Theorem 2.1.

Remark 2.2. When $s=1$, it reduces to interesting relation

$$
\int_{0}^{\infty} B(x, y ; p ; m) d p=B(x+m, y+m)
$$

between the classical and new generalized beta functions, where $\Re e(x+$ $m s)>0, \Re e(y+m s)>0$.

When $m=1$ in (2.5), it reduces to Chaudhry et al. [2] relation.

Remark 2.3. It is interesting to note that all the derivatives of the new generalized beta function with respect to the parameter $p$ can be expressed in terms of the function to give

$$
\begin{aligned}
& \frac{\partial^{n}}{\partial p^{n}}[B(x, y ; p ; m)]=(-1)^{n} B(x-n m, y-n m ; p ; m) \\
& \Re e(p)>0, m>0, \text { and non-negative integer } n .
\end{aligned}
$$

The generalization of Euler's beta function is represented by the various following integral representations. 
192 Dong Myung Lee, Arjun K. Rathie, Rakesh K. Parmar and Yong Sup Kim

\section{Theorem 2.4.}

$$
B(x, y ; p ; m)=2 \int_{0}^{\frac{\pi}{2}} \cos ^{2 x-1} \theta \sin ^{2 y-1} \theta \exp \left[-p \sec ^{2 m} \theta \csc ^{2 m} \theta\right] d \theta ;
$$

$$
B(x, y ; p ; m)=\int_{0}^{\infty} \frac{u^{x-1}}{(1+u)^{x+y}} \exp \left[-p\left(2+u+\frac{1}{u}\right)^{m}\right] d u ;
$$

$$
\begin{aligned}
& B(x, y ; p ; m)=2^{1-x-y} \int_{-1}^{1}(1+u)^{x-1}(1-u)^{y-1} \exp \left[-\frac{4^{m} p}{\left(1-u^{2}\right)^{m}}\right] d u ; \\
& B(x, y ; p ; m)=(c-a)^{1-x-y} \int_{a}^{c}(u-a)^{x-1}(c-u)^{y-1} \\
& \quad \times \exp \left[-p \frac{(c-a)^{2 m}}{(u-a)^{m}(c-u)^{m}}\right] d u, \\
& (2.10) \quad \Re e(p)>0 \text { and } m>0 ; \text { For } p=0, \Re e(x)>0, \Re e(y)>0 ;
\end{aligned}
$$

$$
\begin{aligned}
B(\alpha, \beta ; p ; m)= & 2^{1-\alpha-\beta} \int_{-\infty}^{\infty} \exp \left[(\alpha-\beta) x-4^{m} p \cosh ^{2 m} x\right] \frac{d x}{(\cosh x)^{\alpha+\beta}}, \\
& \Re e(p)>0 \text { and } m>0 ; \text { For } p=0, \Re e(\alpha)>0 \Re e(\beta)>0 .
\end{aligned}
$$

Equations (2.7), (2.8), (2.9), (2.10) and (2.11) can obtain by using the transformations $t=\cos ^{2} \theta, t=\frac{u}{1+u}, t=\frac{1+u}{2}, t=\frac{u-a}{c-a}$ and $t=\tanh x$ in equation (1.13) respectively.

Clearly when $m=1$, equations (2.7) to (2.11) reduce to Chaudhry et al. [2] and when $p=0$, it reduces to usual results for Euler's beta function.

\section{Properties of the new generalized beta function}

We derive the following properties of generalized beta function. To obtain these we first observe that by interchanging the order of summation and integration.

Theorem 3.1.

$$
B(x, y+1 ; p ; m)+B(x+1, y ; p ; m)=B(x, y ; p ; m)
$$


Proof. The left-hand side of (3.1) equals

$$
\int_{0}^{1}\left\{t^{x-1}(1-t)^{y}+t^{x}(1-t)^{y-1}\right\} \exp \left[-\frac{p}{t^{m}(1-t)^{m}}\right] d t
$$

which, after simple algebraic manipulation, yields

$$
\int_{0}^{1} t^{x-1}(1-t)^{y-1} \exp \left[-\frac{p}{t^{m}(1-t)^{m}}\right] d t
$$

which, is equal to the right-hand side of (3.1).

Clearly when $m=1$ equation (3.1) reduces to Chaudhry et al. [2] and when $p=0$, it reduces to usual relation for Euler's beta function [8].

Theorem 3.2. For $\Re(p)>0, \Re(x)>0, \Re(y)<1$ and $m>0$,

$$
B(x, 1-y ; p ; m)=\sum_{n=0}^{\infty} \frac{(y)_{n}}{n !} B(x+n, 1 ; p ; m)
$$

Proof. By the definition of the new generalized beta function equation (1.13), we have

$$
B(x, 1-y ; p ; m)=\int_{0}^{1} t^{x-1}(1-t)^{-y} \exp \left[-\frac{p}{t^{m}(1-t)^{m}}\right] d t .
$$

Using the following binomial series expansion

$$
(1-t)^{-y}=\sum_{n=0}^{\infty}(y)_{n} \frac{t^{n}}{n !}, \quad|t|<1
$$

we obtain

$$
B(x, 1-y ; p ; m)=\int_{0}^{1} \sum_{n=0}^{\infty}(y)_{n} \frac{t^{x+n-1}}{n !} \exp \left[-\frac{p}{t^{m}(1-t)^{m}}\right] d t .
$$

Therefore, interchanging the order of integration and summation and using (1.13), we have 
194 Dong Myung Lee, Arjun K. Rathie, Rakesh K. Parmar and Yong Sup Kim

$$
\begin{aligned}
B(x, 1-y ; p ; m) & =\sum_{n=0}^{\infty} \frac{(y)_{n}}{n !} \int_{0}^{1} t^{x+n-1} \exp \left[-\frac{p}{t^{m}(1-t)^{m}}\right] d t \\
& =\sum_{n=0}^{\infty} \frac{(y)_{n}}{n !} B(x+n, 1 ; p ; m) .
\end{aligned}
$$

Theorem 3.3. For $\Re e(p)>0$ and $m>0$,

$$
B(x, y ; p ; m)=\sum_{n=0}^{\infty} B(x+n, y+1 ; p ; m) .
$$

Proof. Replacing $(1-t)^{y-1}$ in (1.13) by its series representation

$$
(1-t)^{y-1}=(1-t)^{y} \sum_{n=0}^{\infty} t^{n}
$$

we obtain

$$
B(x, y ; p ; m)=\int_{0}^{1}(1-t)^{y} \sum_{n=0}^{\infty} t^{x+n-1} \exp \left[-\frac{p}{t^{m}(1-t)^{m}}\right] d t .
$$

Interchanging the order of integration and summation and using (1.13), we have

$$
\begin{aligned}
B(x, y ; p ; m) & =\sum_{n=0}^{\infty} \int_{0}^{1} t^{x+n-1}(1-t)^{y} \exp \left[-\frac{p}{t^{m}(1-t)^{m}}\right] d t \\
& =\sum_{n=0}^{\infty} B(x+n, y+1 ; p ; m) .
\end{aligned}
$$




\section{Mellin transformation representation of the new general- ized beta function}

In this section, by using the Mellin transform representation of the new generalized beta function in terms of Mellin-Barnes type contour integral, we have the following complex integral representation for $B(x, y$; $p ; m)$ :

Theorem 4.1. For $\Re e(p)>0, m>0$ and $\gamma>0$

$$
B(x, y ; p ; m)=\frac{1}{2 \pi i} \int_{\gamma-i \infty}^{\gamma+i \infty} \frac{\Gamma(s) \Gamma(x+m s) \Gamma(y+m s)}{\Gamma(x+y+2 m s)} p^{-s} d s .
$$

Proof. Taking Mellin transform of (1.13) both sides, we have

$$
\begin{aligned}
& \mathfrak{M}\{B(x, y ; p ; m) ; p \rightarrow s\} \\
& \quad=\int_{0}^{\infty} p^{s-1}\left(\int_{0}^{1} t^{x-1}(1-t)^{y-1} \exp \left[-\frac{p}{t^{m}(1-t)^{m}}\right] d t\right) d p .
\end{aligned}
$$

Changing the order of integration as the integral is uniformly convergent. We have

$$
\begin{aligned}
\mathfrak{M} & \{B(x, y ; p ; m) ; p \rightarrow s\} \\
& =\int_{0}^{1} t^{x-1}(1-t)^{y-1}\left(\int_{0}^{\infty} p^{s-1} \exp \left[-\frac{p}{t^{m}(1-t)^{m}}\right] d p\right) d t .
\end{aligned}
$$

Using the definition of gamma function, we have

$$
\begin{aligned}
\mathfrak{M}\{B(x, y ; p ; m) ; p \rightarrow s\} & =\int_{0}^{1} t^{x-1}(1-t)^{y-1}\left\{t^{m s}(1-t)^{m s} \Gamma(s)\right\} d t . \\
& =\Gamma(s) \int_{0}^{1} t^{x+m s-1}(1-t)^{y+m s-1} d t \\
& =\Gamma(s) B(x+m s, y+m s) \\
& =\Gamma(s) \frac{\Gamma(x+m s) \Gamma(y+m s)}{\Gamma(x+y+2 m s)} .
\end{aligned}
$$

Finally, taking inverse Mellin transform both sides, we have

$$
B(x, y ; p ; m)=\frac{1}{2 \pi i} \int_{\gamma-i \infty}^{\gamma+i \infty} \frac{\Gamma(s) \Gamma(x+m s) \Gamma(y+m s)}{\Gamma(x+y+2 m s)} p^{-s} d s .
$$




\section{The beta distribution for new generalized beta function}

It is expected that there will be many applications of the new generalized beta function, like there in extended beta function. One application that springs to mind is to statistics. For example, the conventional beta distribution can be extended, by using our new generalized beta function, to variables $a$ and $b$ with an infinite range. It appears that such an generalization may be desirable for the project evaluation and review technique used in some special cases.

We define the beta distribution of new generalized beta function by

$$
F(t)= \begin{cases}\frac{1}{B(a, b ; p ; m)} t^{a-1}(1-t)^{b-1} \exp \left[-\frac{p}{t^{m}(1-t)^{m}}\right] & , 0<t<1 \\ 0 & \text {, otherwise. }\end{cases}
$$

If $\nu$ is any real number, then for $p>0,-\infty<a<\infty,-\infty<b<\infty$; $m>0$

$$
E\left(X^{\nu}\right)=\frac{B(a+\nu, b ; p ; m)}{B(a, b ; p ; m)} .
$$

In particular, for $\nu=1$

$$
\mu=E(X)=\frac{B(a+1, b ; p ; m)}{B(a, b ; p ; m)}
$$

represents the mean of the distribution and

$$
\begin{aligned}
\sigma^{2} & =E\left(X^{2}\right)-\{E(X)\}^{2} \\
& =\frac{B(a, b ; p \cdot m) B(a+2, b ; p ; m)-B^{2}(a+1, b ; p ; m)}{B^{2}(a, b ; p ; m)}
\end{aligned}
$$

is the variance of the distribution.

The moment generation function of the distribution is

$$
M(t)=\sum_{n=0}^{\infty} \frac{t^{n}}{n !} E\left(X^{n}\right)=\frac{1}{B(a, b ; p ; m)} \sum_{n=0}^{\infty} B(a+n, b ; p ; m) \frac{t^{n}}{n !} .
$$

The cummulative distribution of (5.1) can be written as

$$
F(X)=\frac{B_{X}(a, b ; p ; m)}{B(a, b ; p ; m)},
$$


where

$$
\begin{aligned}
& B_{X}(a, b ; p ; m)=\int_{0}^{X} t^{a-1}(1-t)^{b-1} \exp \left[-\frac{p}{t^{m}(1-t)^{m}}\right] d t \\
& p>0, \quad-\infty<a<\infty, \quad-\infty<b<\infty ; m>0
\end{aligned}
$$

is new generalized incomplete beta function.

\section{Integral representations of the generalized hypergeomet- ric and confluent hypergeometric function}

In this section we used the new generalization (1.13) of beta functions to generalized the Gauss's hypergeometric function and confluent hypergeometric functions defined by

$$
\begin{aligned}
& F_{p}(a, b ; c ; z ; m)=\sum_{n=0}^{\infty} \frac{B(b+n, c-b ; p ; m)}{B(b, c-b)}(a)_{n} \frac{z^{n}}{n !}, \\
& p \geq 0, \quad m>0, \quad|z|<1 ; \Re e(c)>\Re e(b)>0
\end{aligned}
$$

and

$$
\begin{aligned}
& \Phi_{p}(b ; c ; z ; m)=\sum_{n=0}^{\infty} \frac{B(b+n, c-b ; p ; m)}{B(b, c-b)} \frac{z^{n}}{n !}, \\
& p \geq 0, \quad m>0, \quad \Re e(c)>\Re e(b)>0 .
\end{aligned}
$$

We call the $F_{p}(a, b ; c ; z ; m)$ and $\Phi_{p}(b ; c ; z ; m)$ by the generalized extended Gauss hypergeometric function (GEGHF) and generalized extended confluent hypergeometric function(GECHF) respectively.

The integral representation of GEGHF for $m>0$ is given by using (1.13) and (6.1), we have

$$
\begin{aligned}
& F_{p}(a, b ; c ; z ; m) \\
& =\frac{1}{B(b, c-b)} \int_{0}^{1} t^{b-1}(1-t)^{c-b-1} \\
& \quad \times \exp \left[-\frac{p}{t^{m}(1-t)^{m}}\right] \sum_{n=0}^{\infty}(a)_{n} \frac{(z t)^{n}}{n !} d t, \\
& p>0 ; p=0, m>0 \text { and }|z|<1 ; \Re e(c)>\Re e(b)>0 .
\end{aligned}
$$


198 Dong Myung Lee, Arjun K. Rathie, Rakesh K. Parmar and Yong Sup Kim

Since $(1-z t)^{-a}=\sum_{n=0}^{\infty}(a)_{n} \frac{(z t)^{n}}{n !}$, so we can write $(6.3)$ as

$$
\begin{aligned}
& F_{p}(a, b ; c ; z ; m) \\
& \quad=\frac{1}{B(b, c-b)} \int_{0}^{1} t^{b-1}(1-t)^{c-b-1}(1-z t)^{-a} \exp \left[-\frac{p}{t^{m}(1-t)^{m}}\right] d t, \\
& p>0 ; p=0, m>0 \text { and }|\arg (1-z)|<\pi ; \Re e(c)>\Re e(b)>0 .
\end{aligned}
$$

The another form is obtained by putting $t=\frac{u}{1+u}$ in (6.4), we get

$$
\begin{aligned}
& F_{p}(a, b ; c ; z ; m) \\
& =\frac{1}{B(b, c-b)} \int_{0}^{\infty} u^{b-1}(1+u)^{a-c}[1+u(1-z)]^{-a} \\
& \quad \times \exp \left[-p\left(2+u+\frac{1}{u}\right)^{m}\right] d u, \\
& p>0 ; p=0, m>0 \text { and }|\arg (1-z)|<\pi ; \Re e(c)>\Re e(b)>0 .
\end{aligned}
$$

Also by substituting $t=\sin ^{2} \nu$ in (6.4), we get

$$
\begin{aligned}
& F_{p}(a, b ; c ; z ; m) \\
& =\frac{2}{B(b, c-b)} \int_{0}^{\frac{\pi}{2}} \frac{(\sin \nu)^{2 b-1}(\cos \nu)^{2 c-2 b-1}}{\left(1-z \sin ^{2} \nu\right)^{a}} \\
& \quad \times \exp \left(-p \sec ^{2 m} \nu \csc ^{2 m} \nu\right) d \nu .
\end{aligned}
$$

Further if we substitute $t=\tanh ^{2} \nu$ in (6.4), the another integral representation is obtained as

$$
\begin{aligned}
& F_{p}(a, b ; c ; z ; m) \\
& =\frac{2}{B(b, c-b)} \int_{0}^{\infty} \frac{(\sinh \nu)^{2 b-1}(\cosh \nu)^{2 a-2 c+1}}{\left(\cosh ^{2} \nu-z \sinh ^{2} \nu\right)^{a}} \\
& \quad \times \exp \left(-p \cosh ^{2 m} \nu \operatorname{coth}^{2 m} \nu\right) d \nu .
\end{aligned}
$$

A Mellin-Barnes contour integral representation for $F_{p}(a, b ; c ; z ; m)$ can be obtained by equation (6.4) and the fact that the function $B(x, y ; p ; m)$ has no finite singularities in $x$ and $y$. We thus have 


$$
\begin{aligned}
& F_{p}(a, b ; c ; z ; m) \\
& =\frac{1}{2 \pi i B(b, c-b)} \int_{-i \infty}^{i \infty} \Gamma(-s) \frac{\Gamma(a+s)}{\Gamma(a)} \\
& \quad \times B(b+s, c-b ; p ; m)(-z)^{s} d s .
\end{aligned}
$$

Similarly, the integral representation of GECHF is defined by using (6.2), we have

$$
\begin{aligned}
& \Phi_{p}(b ; c ; z ; m) \\
& \quad=\frac{1}{B(b, c-b)} \int_{0}^{1} t^{b-1}(1-t)^{c-b-1} \exp \left[z t-\frac{p}{t^{m}(1-t)^{m}}\right] d t, \\
& p \geq 0 ; m>0 \text { and } \Re e(c)>\Re e(b)>0 .
\end{aligned}
$$

Also if we replace $t$ by $1-t$, we get another integral representation of (6.9). Thus we have

$$
\begin{aligned}
& \Phi_{p}(b ; c ; z ; m) \\
& \quad=\frac{\exp (z)}{B(b, c-b)} \int_{0}^{1} t^{c-b-1}(1-t)^{b-1} \exp \left[-z t-\frac{p}{t^{m}(1-t)^{m}}\right] d t, \\
& p>0 ; p=0 \text { and } \Re e(c)>\Re e(b)>0 ; m>0 .
\end{aligned}
$$

Clearly for $m=1$ in (6.4) - (6.9) leads to the integral representation of Chaudhry et al. [6] and for $p=0$ in (6.4) - (6.9) leads to integral representation of the usual GHF and CHF [10, pp. 20-23].

\section{Differentiation formulas for the GEGHF and GECHF}

The differentiation formulas for the GEGHF and GECHF can be obtained by differentiating (6.1) and (6.2) with respect to the variable $z$ in terms of a parameter by using the formulas:

$$
B(b, c-b)=\frac{c}{b} B(b+1, c-b)
$$

and

$$
(a)_{n+1}=a(a+1)_{n}
$$


200 Dong Myung Lee, Arjun K. Rathie, Rakesh K. Parmar and Yong Sup Kim

Note for GEGHF, we differentiate (6.1) with respect to $z$, we have

$$
\frac{d}{d z}\left\{F_{p}(a, b ; c ; z ; m)\right\}=\sum_{n=1}^{\infty}(a)_{n} \frac{B(b+n, c-b ; p ; m)}{B(b, c-b)} \frac{z^{n-1}}{(n-1) !} .
$$

Changing $n$ into $n+1$ and using (6.1), we have

$$
\frac{d}{d z}\left\{F_{p}(a, b ; c ; z ; m)\right\}=\frac{a b}{c} F_{p}(a+1, b+1 ; c+1 ; z ; m) .
$$

Recursive application of this procedure gives the general form,

$$
\frac{d^{n}}{d z^{n}}\left\{F_{p}(a, b ; c ; z ; m)\right\}=\frac{(a)_{n}(b)_{n}}{(c)_{n}} F_{p}(a+n, b+n ; c+n ; z ; m) .
$$

A similar procedure for GECHF, we have

$$
\frac{d^{n}}{d z^{n}}\left\{\Phi_{p}(b ; c ; z ; m)\right\}=\frac{(b)_{n}}{(c)_{n}} \Phi_{p}(b+n ; c+n ; z ; m) .
$$

Clearly when $m=1$ in (7.4) and (7.5) leads to the differentiation formulas for Chaudhry et al. [6] and for $p=0$ in (7.4) and (7.5) lead to the differential formulas for usual GHF and CHF [10].

\section{Thw Mellin transform representations of GEGHF and GECHF}

Taking Mellin transform of (6.4), we get Mellin transform of GEGHF as

$$
\begin{aligned}
\mathfrak{M} & \left\{F_{p}(a, b ; c ; z ; m): p \rightarrow s\right\} \\
= & \int_{0}^{\infty} p^{s-1} F_{p}(a, b ; c ; z ; m) d p \\
= & \frac{1}{B(b, c-b)} \int_{0}^{1} t^{b-1}(1-t)^{c-b-1}(1-z t)^{-a} \\
\quad & \times\left(\int_{0}^{\infty} p^{s-1} \exp \left[-\frac{p}{t^{m}(1-t)^{m}}\right] d p\right) d t .
\end{aligned}
$$

Using definition of gamma function in (8.1) and using (1.3), we have 


$$
\begin{aligned}
\mathfrak{M} & \left\{F_{p}(a, b ; c ; z ; m): p \rightarrow s\right\} \\
& =\frac{1}{B(b, c-b)} \int_{0}^{1} t^{b-1}(1-t)^{c-b-1}(1-z t)^{-a}\left\{t^{m s}(1-t)^{m s} \Gamma(s)\right\} d t \\
& =\frac{\Gamma(s)}{B(b, c-b)} \int_{0}^{1} t^{b+m s-1}(1-t)^{c+m s-b-1}(1-z t)^{-a} d t \\
& =\frac{\Gamma(s) B(b+m s, c+m s-b)}{B(b, c-b)}{ }_{2} F_{1}(a, b+m s ; c+2 m s ; z) .
\end{aligned}
$$

Now taking inverse Mellin transforms of both sides of (8.2), we get, an elegant relation between the GEGHF and original function.

$$
\begin{aligned}
F_{p}(a, b ; c ; z ; m)= & \frac{1}{2 \pi i} \int_{\gamma-i \infty}^{\gamma+i \infty} \frac{\Gamma(s) B(b+m s, c+m s-b)}{B(b, c-b)} \\
& \times{ }_{2} F_{1}(a, b+m s ; c+2 m s ; z) p^{-s} d s,
\end{aligned}
$$

where $\gamma>0$. A similar procedure yields us the Mellin transform of GECHF as follows:

$$
\begin{aligned}
\mathfrak{M} & \left\{\Phi_{p}(b, c ; z ; m): p \rightarrow s\right\} \\
& =\frac{\Gamma(s) B(b+m s, c+m s-b)}{B(b, c-b)}{ }_{1} F_{1}(b+m s ; c+2 m s ; z) .
\end{aligned}
$$

Now taking inverse Mellin transform of both side of (8.4), we get

$$
\begin{aligned}
\Phi_{p}(b ; c ; z ; m)= & \frac{1}{2 \pi i} \int_{\gamma-i \infty}^{\gamma+i \infty} \frac{\Gamma(s) B(b+m s, c+m s-b)}{B(b, c-b)} \\
& \times{ }_{1} F_{1}(b+m s ; c+2 m s ; z) p^{-s} d s,
\end{aligned}
$$

where $\gamma>0$.

\section{Transformation formulas for the GEGHF and GECHF}

For the GEGHF, we have the following transformation: 


\section{Theorem 9.1.}

$$
\begin{aligned}
& F_{p}(a, b ; c ; z ; m)=(1-z)^{-a} F_{p}\left(a, c-b ; c ; \frac{z}{z-1} ; m\right) \\
& \text { where }|\arg (1-z)|<\pi .
\end{aligned}
$$

Proof. By writing

$$
[1-z(1-t)]^{-a}=(1-z)^{-a}\left(1+\frac{z}{1-z} t\right)^{-a}
$$

and replacing $t$ by $1-t$ in (6.4), we have

$$
\begin{aligned}
& F_{p}(a, b ; c ; z ; m) \\
& =\frac{(1-z)^{-a}}{B(b, c-b)} \int_{0}^{1} t^{c-b-1}(1-t)^{b-1}\left(1+\frac{z}{1-z} t\right)^{-a} \\
& \quad \times \exp \left[-\frac{p}{t^{m}(1-t)^{m}}\right] d t \\
& p>0 ; p=0 ; m>0 \text { and }|z|<1 ; \Re e(c)>\Re e(b) . \\
& F_{p}(a, b ; c ; z ; m)=(1-z)^{-a} F_{p}\left(a, c-b ; c ; \frac{z}{z-1} ; m\right) \\
& |\arg (1-z)|<\pi .
\end{aligned}
$$

Clearly, when $m=1$ in the transformation formulas (9.2) and (9.3) leads to Chaudhry et al. [6] transformation formulas and for $p=0$ in the transformation formulas (9.2) and (9.3) leads to standard formula for the usual GHF [8, p. 64 Equation 2.1.4 (2)].

Also from (6.9) and (6.10), we can obtain the following transformation formula

\section{Theorem 9.2.}

$$
\Phi_{p}(b ; c ; z ; m)=\exp (z) \Phi_{p}(c-b ; c ;-z ; m) .
$$

Clearly when $m=1$, it reduces to Chaudhry et al. [6] transformation formula and for $p=0$ it reduces to Kummer's first formula [9, p. 125, Theorem 42].

Remark 9.3. Replacing $z$ by $1-\frac{1}{z}$ in (9.3), we get the following transformation formula

$$
F_{p}\left(a, b ; c ; 1-\frac{1}{z} ; m\right)=z^{a} F_{p}(a, c-b ; c ; 1-z ; m), \quad(|\arg (z)|<\pi) .
$$


Remark 9.4. Replacing $z$ by $\frac{z}{1+z}$ in (9.3), we get the following transformation formula

$F_{p}\left(a, b ; c ; \frac{z}{1+z} ; m\right)=(1+z)^{a} F_{p}(a, c-b ; c ; z ; m) \quad(|\arg (1+z)|<\pi)$.

\section{Recurrence relations for the GEGHF and GECHF}

For the GEGHF, we have the following recurrence relation:

Theorem 10.1.

$$
\Delta_{a} F_{p}(a, b ; c ; z ; m)=\frac{b z}{c} F_{p}(a+1, b+1 ; c+1 ; z ; m) .
$$

Proof. By integral representation (6.4) of the GEGHF and if $\Delta_{a}$ is the shift operator with respect to $a$, we see that

$$
\begin{aligned}
\Delta_{a} & F_{p}(a, b ; c ; z ; m) \\
= & F_{p}(a+1, b ; c ; z ; m)-F_{p}(a, b ; c ; z ; m) \\
= & \frac{1}{B(b, c-b)} \int_{0}^{1} t^{b-1}(1-t)^{c-b-1}(1-z t)^{-a-1}\{1-(1-z t)\} \\
& \times \exp \left[-\frac{p}{t^{m}(1-t)^{m}}\right] d t \\
= & \frac{z}{B(b, c-b)} \int_{0}^{1} t^{b}(1-t)^{c-b-1}(1-z t)^{-a-1} \\
& \quad \times \exp \left[-\frac{p}{t^{m}(1-t)^{m}}\right] d t .
\end{aligned}
$$

Changing $a, b$ and $c$ to $a+1, b+1$ and $c+1$ in (6.4), respectively,

$$
\begin{aligned}
& F_{p}(a+1, b+1 ; c+1 ; z ; m) \\
& =\frac{1}{B(b+1, c-b)} \int_{0}^{1} t^{b}(1-t)^{c-b-1}(1-z t)^{-a-1} \\
& \quad \times \exp \left[-\frac{p}{t^{m}(1-t)^{m}}\right] d t .
\end{aligned}
$$


204 Dong Myung Lee, Arjun K. Rathie, Rakesh K. Parmar and Yong Sup Kim

Using the result (7.1) and (10.3) in (10.2), we have

$$
\Delta_{a} F_{p}(a, b ; c ; z ; m)=\frac{b z}{c} F_{p}(a+1, b+1 ; c+1 ; z ; m)
$$

which is our first recurrence formula GEGHF. For GECHF, we have the following recurrence relation:

\section{Theorem 10.2.}

$$
b \Delta_{b} \Phi_{p}(b ; c+1 ; z ; m)+c \Delta_{c} \Phi_{p}(b ; c ; z ; m)=0 .
$$

Now replacing $b$ by $b+1$ and $c$ by $c+1$ in (6.9) and using (7.1), after little simplification, we have

$$
b \Delta_{b} \Phi_{p}(b ; c+1 ; z ; m)+c \Delta_{c} \Phi_{p}(b ; c ; z ; m)=0
$$

which is a recurrence relation for the GECHF where $\Delta_{b}$ and $\Delta_{c}$ are shift operator with respect to $b$ and $c$.

Clearly when $m=1$ (10.6) leads to Chaudhry et al.[6] results and for $p=0$, it reduces to

$$
{ }_{1} F_{1}(b+1, c+1 ; z)=\frac{c}{b}{ }_{1} F_{1}(b, c ; z)+\left(1-\frac{c}{b}\right){ }_{1} F_{1}(b ; c+1 ; z)
$$

Again using the differentiation formula (7.5) with $n=1$, (10.6) can reduces to differential-difference equation form as follows:

$$
\frac{d}{d z}\left\{\Phi_{p}(b ; c ; z ; m)\right\}=\frac{b}{c} \Phi_{p}(b ; c+1 ; z ; m)-\Delta_{c} \Phi_{p}(b ; c ; z ; m)
$$

\section{Summation formula for GEGHF}

We know Gauss's Summation formula [10, p. 2]

$$
\begin{aligned}
{ }_{2} F_{1}(a, b ; c ; 1) & =\frac{\Gamma(c) \Gamma(c-a-b)}{\Gamma(c-a) \Gamma(c-b)} \\
& =\frac{B(b, c-a-b)}{B(b, c-b)}, \quad \Re e(c-a-b)>0
\end{aligned}
$$

Putting $z=1$ in (6.4), we get

$$
\begin{aligned}
& F_{p}(a, b ; c ; 1 ; m)= \\
& \quad \frac{1}{B(b, c-b)} \int_{0}^{1} t^{b-1}(1-t)^{c-a-b-1} \exp \left[-\frac{p}{t^{m}(1-t)^{m}}\right] d t .
\end{aligned}
$$


Now the integral on the right-hand side of (11.2) can be written in term of new generalized beta function. Thus, we have

$$
\begin{aligned}
& F_{p}(a, b ; c ; 1 ; m)=\frac{B(b, c-a-b ; p ; m)}{B(b, c-b)}, \\
& p>0 ; p=0 ; m>0 \text { and } \Re e(c-a-b)>0 .
\end{aligned}
$$

Now for $m=1$, (11.3) leads to Chaudhry et al. [6] and for $p=0$, (11.3) reduces to Gauss's summation formula (11.1).

\section{References}

[1] M. A. Chaudhry, S. M. Zubair, Generalized incomplete gamma function with applications, J. Comput. Appl. Math. 55 (1994), 99-124.

[2] M. A. Chaudhry, A. Qadir, M. Rafique, S. M. Zubair, Extension of Euler's beta function, J. Comput. Appl. Math. 78 (1997), 19-32.

[3] M. A. Chaudhry, S. M. Zubair, On the decomposition of generalized incomplete gamma functions with applications to Fourier transforms, J. Comput. appl. Math. 59 (1995), 253-284

[4] M. A. Chaudhry, N. M. Temme, E. J. M. Veling, Asymptotic and closed form of a generalized incomplete gamma function, J. Comput. Appl. Math. 67 (1996), 371-379.

[5] M. A. Chaudhry, S. M. Zubair, Extended incomplete gamma functions with applications, J. Math. Anal. Appl. 274 (2002), 725-745.

[6] M. A. Chaudhry, A. Qadir, H. M. Srivastava, R. B. Paris, Extended hypergeometric and confluent hypergeometric functions, Appl. Math. Comput, 159 (2004), 589-602.

[7] M. A. Chaudhry, S. M. Zubair, On a class of incomplete gamma functions with applications, CRC Press (Chapman and Hall), Boca Raton, FL, 2002.

[8] A. Erdelyi, W. Magnus, F. Oberhettinger, F. G. Tricomi, Tables of integral transforms, Vol. I, McGraw-Hill, New York, 1954.

[9] E.D. Rainville, Special functions, Macmillan Company, New York, 1960; Reprinted by Chelsea Publishing Company, Bronx, New York, 1971.

[10] L. J. Slater, Generalized hypergeometric functions, Cambridge University Press, Cambridge, 1966.

Dong Myung Lee

Department of Mathematics Education, Wonkwang University, Iksan 570-749, Korea.

E-mail: dmlee@wonkwang.ac.kr 
206 Dong Myung Lee, Arjun K. Rathie, Rakesh K. Parmar and Yong Sup Kim

Arjun K. Rathie

Department of Mathematics, Vedant College of Engineering \& Technology,

TULSI, Distt. Bundi, Rajasthan State, India.

E-mail: akrathie@rediffmail.com

Rakesh K. Parmar

Department of Mathematics, Govt. College of Engineering \& Technology,

Bikaner Rajasthan State, India.

E-mail: rakeshkumarparmar@rediffmail.com

Yong Sup Kim

Department of Mathematics Education, Wonkwang University, Iksan 570-749, Korea.

E-mail: yspkim@wonkwang.ac.kr 